\title{
Are the Quality and Safety of Magistral Preparations Guaranteed Enough?
}

\section{Paola Minghetti*, Laura C. Romanazzi, Antonella Casiraghi and Francesca Selmin}

Department of Pharmaceutical Sciences, Universitàdegli Studi di Milano, Italy

Prescription of medicinal products constitutes one of the main activities of clinical practice with the primary purpose to assure the most advantageous treatment in case of particular disease. Due to rapid development of medical science and technologies, regulatory Authorities have continuously moved to implement new legislation and increase data required to grant a marketing authorization (MA) and assure quality, efficacy and safety of medicinal products.

However, approved medicines currently on the market do not address all public health therapeutic needs. Indeed, drugs for rare pathologies and dosage form suitable for a particular administration site or age-appropriate dosage forma doses formulated at different strength with safe excipients are not available. As matter of fact, MA of orphan drug is inhibited by the existence of often limited and weak clinical data at launch time. In the context of rare disease, it may prove difficult to recruit a sufficient number of patients and medical centers in clinical trials [1-3]. For many decades, also children were considered "therapeutic orphan" because of lack of clinical data and ethical concerns ruling out young patients; hence $67 \%$ of paediatric population received an unlicensed or off-label prescription.

When authorized medicines are not available or do not meet the specific needs of the patients, the physician can resort to prescribing compound medicines. Pharmacists are the only healthcare professionals formally trained in the art of compounding, thus the skills of the compounding pharmacists are needed to satisfy the individualized needs. Therefore, the pharmacist both in hospitals and community pharmacies has the duty to compound the extemporaneous medicine taking responsibility for ensuring that is of suitable quality, safe, stable and effective.

The European Pharmacopoeia contains a monograph about "Pharmaceutical preparations" which allows supplying unlicensed products extemporaneously prepared for a specific patient or made in larger batches for stock keeping. Despite the efforts made to assure the same level of quality and safety of industrial products, range of challenging issues remains. Indeed, safety and effectiveness of a drug product depends on the potency, purity and quality of ingredients, which in turn can be affected by the process of extemporaneous preparation. Risks in compounding are associated to errors of dose calculations or measurements, the use of incorrect formulae with no information of chemical, physical or microbiological stability, wrong method in compounding, incompatibility among ingredients and/or container and limited skills of pharmacists [4-6].

The regulation concerning the extemporaneous preparations in pharmacies falls under the national policies of each single State of the European Community and are not harmonised throughout Europe.

Over years, the competent Authorities have issued guides on pharmaceutical preparations with the intent to guarantee the safety and quality and to protect public health.

Following the structure from the Good Manufacturing PracticesGuide for industry, the PIC/S Guide was [7] lay out guidance on Good Practices (called Good Preparation Practice-GPP) on the preparation of medicinal products for human use only. This Guide examines how the potential risk for health damage in case of failure varies with different types of products and should therefore be assessed and documented by an appropriately competent person.
Examples are also provided to evidence how the potential risk could be mainly influenced by the probability of occurrence of a mistake, probability of detection of a possible mistake and, more important, the consequence on health of a possible mistake (health risk).

With a view to avoiding quality and safety gaps between extemporaneous and manufactured products, the Committee of Minister of the Council of Europe issued a resolution [8], recommended the introducing of two fundamental key concepts: the added value of all pharmacy preparations for humans and the risk assessment.

Medicinal products compounded in pharmacies, both extemporaneous and for stock are of added value whether they are needed by a specific patient or specific population groups with particular needs. Nevertheless, if a suitable pharmaceutical equivalent with a MA is available, the preparation should not be dispensed. Moreover, the professional involved in patient care should jointly undertake an appropriate assessment of the risk for the patient, which includes the risk posed by unlicensed medicinal product and the risk related to the unavailability of this medicinal product.

The Resolution referred a risk-based decision matrix to individuate "high-risk preparations" and "low-risk-preparations". The matrix is based on type of preparation; amount prepared annually, pharmacological effect of the active substances, preparation process and supply.

The Resolution recommended using the GMP Guide as a reference for an appropriate quality system for "high-risk preparations", and the GPP Guide for "low-risk-preparations". The application of other guidelines with an equivalent quality level is also accepted, depending on national legislation or guidance.

It is worthy to underline that this Resolution was the first at Community level, to introduce a simple criterion to classify preparations in two classes on the bases of the assessed risk and to guarantee the good level of quality.

Nevertheless, the current situation remains blurred since not all pharmacists are aware of these guides or evaluate the added value or apply the risk-based decision matrix to individuate the level of the quality system required for the pharmacy preparation process.

Finding a solution of these issues is one of the main challenges faced by the national authorities in the next years. Technical data, more frequently compounded formulations and a decision tree could be shared between scientific association and technical body (e.g.

*Corresponding author: Paola Minghetti, Department of Pharmaceutical Sciences, Universitàdegli Studi di, Milano, Via G. Colombo, 71 - 20133 Milano, Italy, Tel: +39 02 50324639 ; Fax +39 02503 24657; E-mail: paola.minghetti@unimi.it

Received February 04, 2014; Accepted February 05, 2014; Published February 15,2014

Citation: Minghetti P, Romanazzi LC, Casiraghi A, Selmin F (2014) Are the Quality and Safety of Magistral Preparations Guaranteed Enough? Pharmaceut Reg Affairs 3: e126. doi:10.4172/2167-7689.1000e126

Copyright: ( $) 2014$ Minghetti $P$, et al. This is an open-access article distributed under the terms of the Creative Commons Attribution License, which permits unrestricted use, distribution, and reproduction in any medium, provided the original author and source are credited. 
Pharmacopeia Commission) in order to support professionals in the decision-making process, namely the prescriber and the pharmacist.

The competent authorities should also make an effort to harmonise rules in Europe aiming to protect the patient and public health and to guarantee the suitable standard of quality and safety of magistral preparations.

\section{References}

1. Woodfield T (1999) Children and research: ethical and philosophical principles. Paediatr Nurs 11: 36-39.

2. Lamprill J (2002) Asking for children's assent to take part in clinical research Good ClinPract.

3. European Medicines Agency.Topic E11Notes for guidance on the clinical investigation of paediatric medicinal products in the paediatric population. Document number CPMP/ICH/2711/99. London: European Medicines Agency.
4. Neubert A, Wong IC, Bonifazi A, Catapano M, Felisi M, et al. (2008) Defining off-label and unlicensed use of medicines for children: results of a Delphi survey. Pharmacol Res 58: 316-322.

5. European Pharmacopoeia (edn 8th).

6. Minghetti P, Palmieri I, Selmin F (2010) When authorized medicinal products are not available: possible alternatives to meet legitimate expectations of patients. Journal of Pharmaceutical Health Services Research 1: 107-112.

7. Good preparation practices (PIC/S GPP): "Guide to good practices for the preparation of medicinal products in healthcare establishment", in Pharmaceutical Inspection Convention Pharmaceutical Inspection (PIC/S) Guide PE010.

8. Resolution CM/Res AP (2011)1 on quality and safety assurance requirements for medicinal products prepared in pharmacies for the special need of patients, as adopted by the Committee of Ministers on 19 January 2011. 